\title{
Canine cognitive dysfunction patients have reduced total hippocampal volume compared with aging control dogs: A comparative magnetic resonance imaging study
}

\author{
Curtis Wells Dewey ${ }^{1,2,3^{*}}$, Mark Rishniw ${ }^{1}$, Philippa J. Johnson ${ }^{1}$, Simon Platt ${ }^{4}$, Kelsey Robinson ${ }^{4}$, Joseph Sackman ${ }^{2}$ and \\ Marissa O’Donnell ${ }^{2}$ \\ ${ }^{1}$ Department of Clinical Sciences, College of Veterinary Medicine, Cornell University, Ithaca, NY, USA \\ ${ }^{2}$ Department of Surgery, Long Island Veterinary Specialists, Plainview, NY, USA \\ ${ }^{3}$ Rochester Veterinary Specialists and Emergency Services, College of Veterinary Medicine, University of Georgia, \\ Athens, GA, USA \\ ${ }^{4}$ Department of Small Animal Medicine and Surgery, College of Veterinary Medicine, University of Georgia, Athens, GA, USA
}

\begin{abstract}
Background: Hippocampal atrophy is a key pathologic and magnetic resonance imaging (MRI) feature of human Alzheimer's disease (AD). Hippocampal atrophy has not been documented via MRI in canine cognitive dysfunction (CCD), which is considered as the dog model of human AD.

Aim: The purpose of this retrospective comparative volumetric MRI study was to compare total hippocampal volumes between successfully aging (control) dogs and dogs diagnosed with CCD.

Methods: Mimics ${ }^{\circledR}$ software was used to derive total hippocampal volumes and total brain volumes from the MRI studies of 42 aging dogs ( $\geq 9$ years): 16 dogs diagnosed with CCD and 26 successfully aging controls. Hippocampal volumes were normalized to total brain volume and these values were compared between groups using Mann-Whitney U tests.

Results: Total hippocampal volume normalized to total brain volume was significantly less for CCD patients compared with control dogs $(p=0.04)$.

Conclusion: The results of this study suggest that - similar to human AD - hippocampal atrophy is a pathological feature of CCD. This finding has potential importance for both investigating disease mechanisms related to dementia as well as future hippocampal-targeted therapies.
\end{abstract}

Keywords: Canine, Cognitive, Dog, Hippocampal, Volume.

\section{Introduction}

Alzheimer's disease (AD), a degenerative brain disorder of people, shares many clinical and pathological features with canine cognitive dysfunction (CCD), a disorder affecting aging dogs. Consequently, investigators consider CCD a naturally occurring model for studying human AD. Furthermore, CCD commonly causes frustration for dog owners and veterinarians (Chapagain et al., 2018; Dewey et al., 2019). Studies have documented hippocampal damage as an early and prominent pathologic feature in both $\mathrm{AD}$ and CCD and attributed this pathology to the deposition of neurotoxic compounds such as beta-amyloid and tau proteins (Schmidt et al., 2015; Wang et al., 2015; Halliday, 2017). In humans, volumetric measurement of the hippocampi from magnetic resonance imaging (MRI) allows clinicians to assess the presence or absence of hippocampal atrophy, as a diagnostic marker for AD (Schroder and Pantel, 2016). Additionally, MRI-based hippocampal volumetric measurements are being evaluated as means of assessing responses to future hippocampal-based treatment options for AD (Dhikav et al., 2017; Bayram et al., 2018).

The use of MRI to assess hippocampal volume in dogs with CCD has not been reported. The purpose of this MRI study was to compare total hippocampal volumes between dogs with CCD and similarly aged control dogs. We hypothesized that dogs with CCD would have smaller total hippocampal volumes compared with controls.

\section{Materials and Methods}

We searched MRI databases from five institutions (Cornell University Hospital for Animals, University of Georgia, Long Island Veterinary Specialists, Veterinary Specialty and Emergency Services of Rochester, and Oradell Animal Hospital) for brain MRI scans of aging ( $\geq 9$ years old) dogs diagnosed with CCD and similarly aged dogs with no evidence of CCD (controls). Control dogs had undergone MRI for reasons unrelated to CCD, including peripheral vestibular dysfunction, late onset epilepsy, Horner's syndrome, and blindness. 
Because relatively few aged dogs undergo cranial MRI scans for non-central nervous system (CNS) disorders, we expanded our control group by acquiring additional control MRI scans from two sources: 10 mixed breed retired sled dogs with normal neurologic examinations that had been imaged as part of another study and six neurologically normal small breed dogs whose owners volunteered for a no-cost brain MRI prior to scheduled dentistry procedures. Because of the nature of this study, the need for Institutional Animal Care and Use Committee (IACUC) approval was waived by Cornell University's Institute for Animal Care and Use Committee.

We based our diagnosis of CCD on previously established historical and clinical criteria together with characteristic MRI abnormalities (excluding hippocampal measurements) (Hasegawa et al., 2005; Schutt et al., 2015; Dewey et al., 2019). In addition, we only included cases of CCD for which this diagnosis was clearly stated in the medical record and supported by the MRI report.

All MRIs were performed under general anesthesia with one of six magnets: (1) $1.5 \mathrm{~T}$ Siemens Avanto (Munich, Germany), (2) $1.5 \mathrm{~T}$ Toshiba Vantage Elan (Lake Forest, CA), (3) 3.0 T Philips Achieva (Nutley, NJ), or (4) 3.0 T GE Discovery MR750 (Chicago, IL). Imaging sequences acquired included the following: sagittal T2-weighted; transverse T2- and T1-weighted; transverse and dorsal plane T1-weighted postgadolinium injection; transverse T2-fluid attenuated inversion recovery; and transverse $\mathrm{T} 2 *$ gradientrecalled echo. For the 1.5-T MRI units, measurement parameters were as follows: slice thickness, $3.5 \mathrm{~mm}$; slice gap, $3.5 \mathrm{~mm}$; field of view (FOV), $185 \mathrm{~mm}$; matrix size of images, $480 \times 480$. For the $3.0-\mathrm{T}$ MRI units, measurement parameters were as follows: slice thickness, $2.0 \mathrm{~mm}$; slice gap, 1.0-3.0 mm (depending on dog size); FOV, 1,101 mm; matrix size of images, $400 \times 400$.

For each dog, three-dimensional volumes were measured from T2-weighted brain images using Mimics ${ }^{\circledR}$ software by two observers (Joseph Sackman

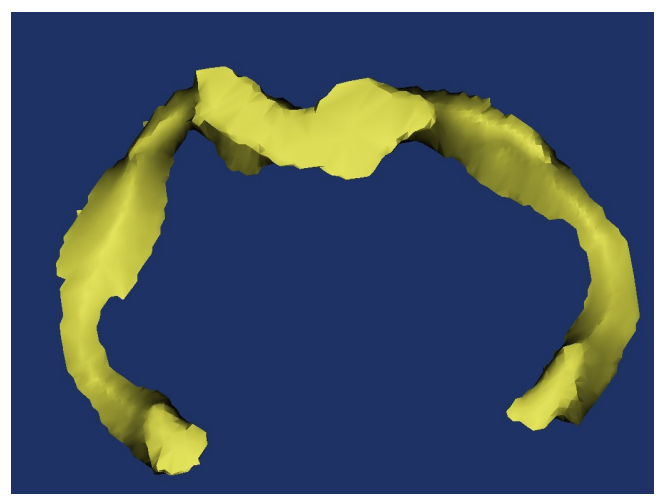

(JS) and Marissa O'Donnell (MO)) who were unaware of the status of the dogs in the study. Quantitative volumetric measurements of both hippocampi as well as total brain volume were acquired for each dog, as previously described (Fig. 1) (Estey et al., 2017). Anatomic landmarks for measurements were used from published reference information (Leigh et al., 2008; Milne et al., 2013).

Wethennormalizedtotalhippocampalvolumestototalbrain volume (rather than bodyweight) under the assumption that total brain volume would not change with $\mathrm{CCD}$, and that total brain volume (but not bodyweight) remains unaffected by body condition (i.e. obesity, emaciation) according to the following equation: $n C O L H I P P=$ Hippocampal VolumeTotalBrainVolume*1000

Because hippocampal volume represents a small percentage of total brain volume, we multiplied the volume ratio by 1,000 to have more easily understood values.

\section{Statistical analyses}

We compared all continuous variables (i.e., normalized hippocampal volumes and age) between the CCD dogs and control dogs using Mann-Whitney U tests.

To assess both intra- and inter-observer variabilities in measurements, 20 patient scans were remeasured by both observers (JS and MO), neither of whom were aware of the patient status (CCD vs control). Agreement between repeated measurements was examined using limits of agreement analysis (Bland and Altman, 1999). Ethical approval

The need for IACUC approval for this investigation was waived by Cornell University’s IACUC committee.

\section{Results}

We included 16 dogs with CCD and 26 control dogs in the study. Dogs with CCD were older than control dogs (median age $13 v v_{.} 11.5$ years; $p=0.0002$ ); however, we could detect no negative correlation between advancing age and reduced hippocampal volume in either group (Fig. 2). The CCD group comprised two Shih Tzus, two Springer spaniels, and one each of Chihuahua, Miniature Poodle, Wheaten terrier, Labrador retriever, Tibetan

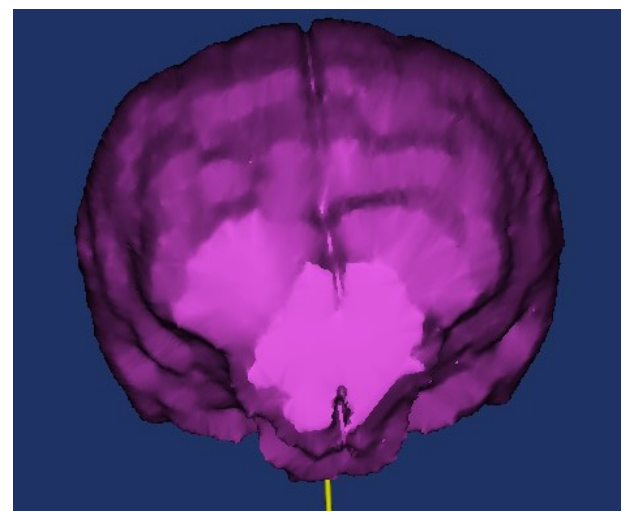

Fig. 1. Examples of hippocampal volume (A) and total brain volume (B) procurement from MR images using Mimics ${ }^{\circledR}$ software. 
terrier, Samoyed, Miniature Schnauzer, Cockapoo, German Shepherd, Shetland Sheepdog, Beagle, and mixed breed. These consisted of nine spayed females, five neutered, and two intact males. The control group comprised 12 mixed breed dogs, four Chihuahuas, and one each of Maltese, Boston terrier, Yorkshire terrier, Miniature Dachshund, Coonhound, Golden Retriever, West Highland White terrier, Beagle, Havanese, and English Cocker Spaniel. These consisted of 13 spayed and two intact females, seven neutered, and four intact males.

Dogs with CCD had smaller normalized hippocampal volumes than control dogs (median 6.2 vs. 7.9; $p=0.04$; Fig. 3). Limits of agreement analysis showed repeat measurements to agree with original measurements within a $30 \%$ range.

\section{Discussion}

Our study demonstrates that dogs afflicted with CCD have smaller hippocampal volumes compared to similarly aged control dogs without CCD. The differences were small, and showed a substantial degree of overlap between the two groups, suggesting that hippocampal volumetric measurements in dogs will be unable to discriminate between healthy and CCD dogs on an individual basis. However, our data suggest that hippocampal atrophy is not part of canine aging, as we could find no clear association between age and hippocampal volumes in either group.

Hippocampal atrophy is a key and early feature of human $\mathrm{AD}$, and loss of hippocampal neurons and synapses is strongly associated with cognitive decline in that disorder (Wang et al., 2015; Halliday, 2017; Ferrari et al., 2019). Hippocampal pathology has been documented in brains of dogs with CCD, but hippocampal atrophy has not been demonstrated for this disorder (Schmidt et al., 2015). In addition to hippocampal atrophy being a central pathophysiologic aspect of $\mathrm{AD}$, the hippocampus is a source of neuronal stem and progenitor cells. Research

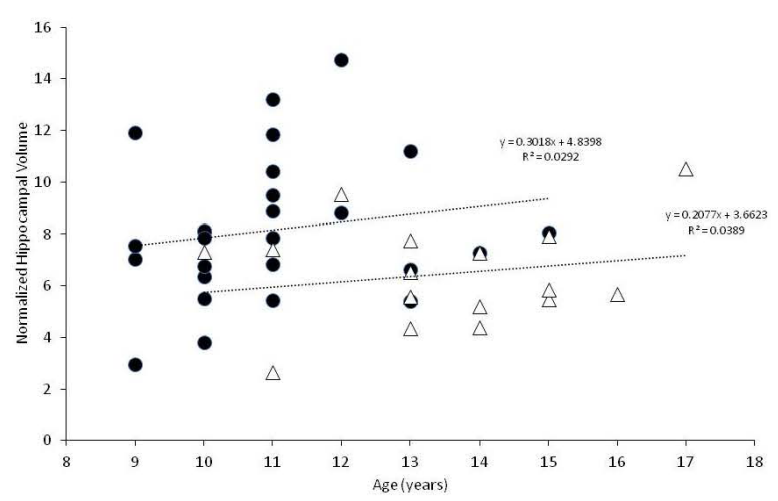

Fig. 2. Normalized hippocampal volumes vs. age for controls (black circles) and CCD dogs (open triangles). Linear regression analysis is provided for each group. into the roles of the hippocampus in $\mathrm{AD}$ pathogenesis and potential methods of positively affecting hippocampal function to treat $\mathrm{AD}$ patients is ongoing (Kirschen and Ge, 2019). CCD is considered a naturally occurring canine analogue of human AD (Hasegawa et al., 2005; Schmidt et al., 2015; Schutt et al., 2015; Chapagain et al., 2018; Dewey et al., 2019). Hippocampal-directed research in CCD patients may potentially benefit dogs with that disorder and human AD patients.

There are several limitations to this study, most of which are related to its retrospective nature. Although multiple institutions were involved in recruiting case material, the case numbers are still small. Also, the MR images evaluated were derived from multiple different machines, which could introduce some level of variability in the resultant data. We restricted case enrollment to dogs 9 years and older, in accordance with previous publications dealing with aging dogs (Tapp et al., 2004; Schutt et al., 2015; Gardini et al., 2019). Although the median ages of our CCD and control groups was not large (11.5 vs. 13 years), it was statistically significant. A major hurdle in this investigation was locating control MRIs for comparison, most likely due to the low likelihood of dog owners pursuing brain MRIs for very old dogs without evidence of neurologic impairment. The possibility exists that the smaller hippocampal volumes in our CCD group were due to this group being older than the control dogs $v s$. a sequela to a degenerative brain disorder. The authors consider this unlikely for several reasons. Graphic representation (Fig. 2) of hippocampal volumes vs. age does not support a decreasing volume with aging for either the control or CCD groups. In addition, statistical analysis of these data failed to discern a negative correlation between advancing age and hippocampal volume in either group. Age-related hippocampal atrophy has been documented to occur as an aging change in dogs, when young dogs are compared with older dogs (Tapp et al., 2004; Gardini et al., 2019). In one study, linear

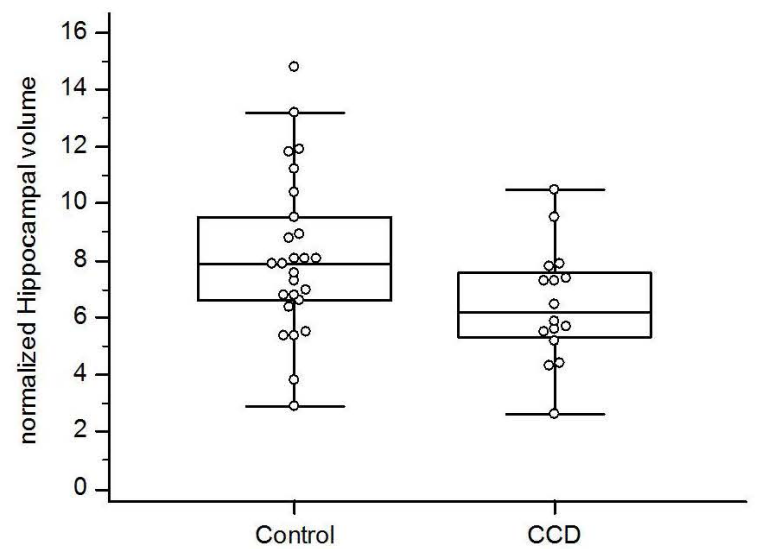

Fig. 3. Box and whisker plots comparing normalized hippocampal volumes between aging control dogs and dogs with CCD. 
MRI measurements of the hippocampi normalized to brain height were compared between young (1-3 years old) and older ( $>10$ years old) dogs with normal brain anatomy; a significant reduction of $2.64 \%$ was found between young and old dogs in that study (Gardini et al., 2019). Although the results of our volumetric study of older dogs are not directly comparable to the results of the linear MRI study, the percentage difference between our two groups of dogs was $21.5 \%$. In a study of laboratory Beagle dogs, hippocampal volumes were compared between young and old dogs using MRI. The older dogs were subdivided into two categories: old dogs (aged 8-11 years) and senior dogs (aged 12 years and older). Although hippocampal volume was shown to decrease when older dogs were compared to younger dogs ( $<8$ years of age), there was no difference in hippocampal volume between the old and senior dog groups (Tapp et al., 2004). In other words, agerelated hippocampal atrophy appears not to progress dramatically as a non-specific aging change in normal dogs over 8 years of age, based on the results of the Beagle study.

Future MRI investigations into hippocampal atrophy in dogs with CCD would benefit from prospective investigations with larger case numbers, more closely age-matched controls, more consistent imaging procurement (i.e., one machine model), and more structurally detailed images (e.g., diffusion tensor imaging and tractography). Additionally, comparison of linear MRI measurements of hippocampal volumes between CCD patients and successfully aging dogs should be performed. Hopefully, investigations into hippocampal-related aspects of $\mathrm{AD}$ will benefit from CCD dogs as a disease model.

In conclusion, we demonstrated that dogs with CCD have significantly smaller total hippocampal volumes, as measured on MR images, compared with successfully aging controls. This finding may have implications in pathophysiologic and therapeutic research into hippocampal-associated aspects of CCD and AD.

Conflict of interest

The authors declare that there is no conflict of interest. Authors' contributions

Drs. Dewey, Rishniw, and Platt were involved with the study inception and design. Dr. Rishniw performed statistical analyses. Dr. Johnson was involved in image analysis and study design. Dr. Robinson was involved in case material acquisition and manuscript preparation. Mr. Sackman and Ms. O'Donnell were involved in case material acquisition and volumetric measurements. All authors were involved in manuscript preparation.

\section{References}

Bayram, E., Caldwell, J.Z.K. and Banks, S.J. 2018. Current understanding of magnetic resonance imaging biomarkers and memory in Alzheimer's disease. Alzheimers Dement. 4, 395-413.
Bland, J.M. and Altman, D.G. 1999. Measuring agreement in method comparison studies. Stat. Methods Med. Res. 8, 136-160.

Chapagain, D., Range, F., Huber, L. and Virányi, Z. 2018. Cognitive aging in dogs. Gerontology 64, 165-171.

Dewey, C.W., Davies, E.S., Xie, H. and Wakshlag, J.J. 2019. Canine cognitive dysfunction: pathophysiology, diagnosis and treatment. Vet. Clin. North Am. Small. Anim. Pract. 49(3), 477-499.Dhikav, V., Duraiswamy, S. and Anand, K.S. 2017. Correlation between hippocampal volumes and medial temporal lobe atrophy in patients with Alzheimer's disease. Ann. Indian Acad. Neurol. 20, 29-35.

Estey, C.M., Dewey, C.W., Rishniw, M., Lin, D.M., Bouma, J., Sackman, J. and Burkland, E. 2017. A subset of dogs with presumptive idiopathic epilepsy show hippocampal asymmetry: a volumetric comparison with non-epileptic dogs using MRI. Front. Vet. Sci. 4, 183; doi:10.3389/fvets.2017.00183

Ferrari, B.L., Campos Neto, G., Nucci, M.P., Mamani, J.B., Lacerda, S.S., Felício, A.C., Amaro, E. and Gamarra, L.F. 2019. The accuracy of hippocampal volumetry and glucose metabolism for the diagnosis of patients with suspected Alzheimer's disease, using automatic quantitative clinical tools. Medicine (Baltimore) 98(45), e17824; doi: 10.1097/ MD.0000000000017824

Gardini, A., Taeymans, O., Cherubini, G.B., de Stefani, A., Targett, M. and Vettorato, E. 2019. Linear magnetic resonance imaging measurements of the hippocampal formation differ in young versus old dogs. Vet. Rec. 185(10), 306.

Halliday, G. 2017. Pathology and hippocampal atrophy in Alzheimer's disease. Lancet Neurol. 16(11), 862-864.

Hasegawa, D., Yayoshi, N., Fujita, Y. and Orima, H. 2005. Measurement of interthalamic adhesion thickness as a criteria for brain atrophy in dogs with and without cognitive dysfunction (dementia). Vet. Radiol. Ultrasound 46, 452-457.

Kirschen, G.W. and Ge, S. 2019. Young at heart: insights into hippocampal neurogenesis in the aged brain. Behav. Brain Res. 369, 111934; doi: 10.1016/j.bbr.2019.111934.

Leigh, E.J., Mackillop, E., Robertson, I.D. and Hudson, L.C. 2008. Clinical anatomy of the canine brain using magnetic resonance imaging. Vet. Radiol. Ultrasound 49, 113-121.

Milne, M.E., Anderson, G.A., Chow, K.E., O’Brien, T.J., Moffat, B.A. and Long, S.N. 2013. Description of technique and lower reference limit for magnetic resonance imaging of hippocampal volumetry in dogs. Am. J. Vet. Res. 74, 224-231.

Schmidt, F., Boltze, J., Jager, C., Hofmann, S., Willems, N., Seeger, J., Härtig, W. and Stolzing, A. 2015. Detection and quantification of $\beta$-amyloid, 
pyroglutamyl $A \beta$, and tau in aged canines. $J$. Neuropath. Exp. Neurol. 74, 912-923.

Schroder, J. and Pantel, J. 2016. Neuroimaging of hippocampal atrophy in early recognition of Alzheimer's disease-a critical appraisal after two decades of research. Psychiatry Res. Neuroimaging 247, 71-78.

Schutt, T., Toft, N. and Berendt, M. 2015. Cognitive dysfunction, progression of age-related behavioral changes, biomarkers, and survival in dogs more than 8 years old. J. Vet. Intern. Med. 29, 1569-1577.
Tapp, P.D., Siwak, C.T., Gao, F.Q., Chiou, J.-Y., Black, S.E., Head, E., Muggenburg, B.A., Cotman, C.W., Milgram, N.W. and Su, M-Y. 2004. Frontal lobe volume, function, and $\beta$-amyloid pathology in a canine model of aging. J. Neurosci. 24, 8205-8213.

Wang, L., Benzinger, T.L., Hassenstab, J., Blazey, T., Owen, C., Liu, J., Fagan, A.M., Morris, J.C. and Ances, B.M. 2015. Spatially distinct atrophy is linked to $ß$-amyloid and tau in preclinical Alzheimer disease. Neurology. 85, 1254-1260. 